\title{
3 Zugang zu Land und Macht - Ein Analyserahmen für Konflikte im Kontext von land grabbing
}

Um analysieren zu können, welcher Zusammenhang zwischen dem Handeln traditioneller Autoritäten im Kontext von land grabbing und Konflikten um Zugang zu Land besteht, kombiniere ich ein theoretisch begründetes Verständnis von Zugang zu Land in traditionellen Landverwaltungssystemen mit einem Machtkonzept, das auf die empirische Analyse ausgerichtet ist. Das Verständnis der Mechanismen, über die unterschiedliche Akteure Zugang zu Land in traditionellen Landverwaltungssystemen erlangen, strukturiert meine Analyse. Es dient dazu, die unterschiedlichen Interessen der Akteure zu erfassen, entlang derer Konflikte um Zugang zu Land verlaufen. Für die Analyse fehlt jedoch ein erklärendes Element, das erlaubt zu untersuchen, wie sich das Handeln von Konfliktakteuren - und insbesondere das Handeln traditioneller Autoritäten - auf den Zugang zu Land auswirkt, und woher sich die Fähigkeit, Zugang zu Land zu kontrollieren, ableitet. Diesem Zweck dient das empirisch nutzbare Konzept von Macht.

Zugang verstehe ich in Anlehnung an Jesse C. Ribot und Nancy Lee Peluso (2003) als die Fähigkeit, einen Nutzen aus etwas ziehen zu können. Zugang $\mathrm{zu}$ Land bedeutet folglich, einen Nutzen aus dem Land ziehen zu können. Beispiele dafür sind die Bestellung des Lands für den Eigenbedarf oder für den Verkauf, die Verpachtung des Lands gegen Geld- oder Naturalleistungen, Lohnarbeit auf dem Land oder die Weiterverarbeitung von auf dem Land befindlichen Ressourcen. Einen Nutzen aus dem Land zu ziehen bedeutet also, über das Land etwas zu gewinnen, das weiterverwertet werden kann. Im Vergleich zu klassischen Debatten um Eigentum - definiert als »das Recht einen Nutzen aus etwas ziehen zu können« (Ribot/Peluso 2003: 154, Übersetzung durch die Autorin) - ermöglicht das theoretische Konzept des Zugangs eine 
umfassendere Analyse. Statt nur auf Eigentums- und Besitzverhältnisse, lenkt es den Blick auf ein breiteres Spektrum sozialer Verhältnisse, die es Menschen ermöglichen oder sie daran hindern, einen Nutzen aus Land zu ziehen. Damit eignet es sich insbesondere für Analysen im Kontext traditioneller Landverwaltungssysteme. In diesen ist Zugang zu Land nicht primär durch staatliches Recht, das formalisiertes Eigentum schafft, bestimmt, sondern durch eine Vielzahl unterschiedlicher Zugangsmechanismen (vgl. Boone 2015). Um Zugang zu Land analytisch greifbar zu machen, arbeite ich, in Anlehnung an Ribot und Peluso (2003) sowie aufbauend auf Literatur zu traditionellen Landrechtssystemen (Amanor 1999; Berry 1989, 1993; Juul/Lund 2002a; Lund 2011, 2013; Peters 1984, 2004; Shipton/Goheen 1992; Sikor/Lund 2009a) zentrale Mechanismen heraus, die bestimmen wer in traditionellen Landverwaltungssystemen wie und wann in der Lage ist, einen Nutzen aus Land zu ziehen (Ribot/Peluso 2003: 154, Hervorhebung im Original). Unter Berücksichtigung der Literatur zu traditionellen Landrechtssystemen leite ich aus dem Beitrag von Ribot und Peluso zwei Varianten von Mechanismen ab, die als relevant für den Zugang zu Land in traditionellen Landverwaltungssystemen gelten: rechtebasierte sowie relationale und strukturelle Mechanismen.

Studien zu Konflikten um Land im Kontext von land grabbing zeigen, dass Zugang zu Land ein relevanter Konfliktgegenstand ist, der oftmals im Zentrum der Konflikte steht (Peluso/Lund 2011; White et al. 2012). In Konflikten oder Kontexten struktureller Veränderungen können nicht alle Akteure in gleichem Maße zuvor bestehenden Zugang zu Land aufrechterhalten, da sie Zugang zu Land nicht gleichberechtigt kontrollieren. Aus diesem Gedanken leite ich die Annahme ab, dass Kontrolle ein zentraler Aspekt für die Analyse von Konflikten um Zugang zu Land ist. In traditionellen Landverwaltungssystemen obliegt die Kontrolle des Zugangs insbesondere - aber nicht ausschließlich - traditionellen Institutionen. Im Fall von chieftaincy-Systemen fordern je nach Kontext unterschiedliche traditionelle Autoritäten das Recht Zugang zu Land zu kontrollieren für sich ein. Um zu verstehen, welcher $\mathrm{Zu}$ sammenhang zwischen dem Handeln traditioneller Autoritäten und Konflikten um Land besteht, ist es daher wichtig zu analysieren, wie traditionelle Autoritäten Zugang zu Land kontrollieren. Haripriya Rangan bezeichnet Kontrolle als die Fähigkeit, das Handeln anderer zu überwachen, zu lenken oder zu regulieren (1997: 72). Entsprechend üben diejenigen Akteure Kontrolle über den Zugang zu Land aus, die in der Lage sind, zu überwachen, zu lenken und zu regulieren, wer einen Nutzen aus Land ziehen kann. Die Fähigkeit den Zugang zu Land zu kontrollieren, wird wiederum als Ausdruck 
von Macht verstanden (Bryant/Bailey 1997; Ribot/Peluso 2003). Obwohl zahlreiche Beiträge zu Konflikten um Zugang zu Land die analytische Relevanz von Macht betonen (vgl. Bavinck et al. 2014; Peluso/Lund 2011; Ribot/Peluso 2003; Sikor/Lund 2009a), bleiben sie bezüglich folgender drei Fragen vage: (1) Welche Formen kann Macht in Konflikten um Zugang zu Land annehmen? (2) Woher leitet sie sich ab? Und (3) wie können Machtverhältnisse in diesem Kontext analytisch in den Blick genommen werden? Diesen Schwachpunkten widme ich mich in meinem Analyserahmen, indem ich ein differenziertes Verständnis von Zugang zu Land in traditionellen Landrechtssystemen mit einem empirisch nutzbaren Konzept von Macht verknüpfe. Macht verstehe ich als relational, was bedeutet, dass Macht nicht absolut ist, sondern in Verhältnissen zwischen Akteuren besteht und diese gestaltet. Als einen für die empirische Analyse nutzbaren Ansatz zur Untersuchung von Machtverhältnissen integriere ich Steven Lukes' Konzept der drei Dimensionen von Macht in meinen Analyserahmen (Lukes 2005). Mit Blick auf die empirische Analyse bietet es gegenüber anderen Machtkonzepten einige entscheidende Vorteile. Lukes greift zentrale Uneinigkeiten und Widersprüche bezüglich der Konzeption von Macht - die in unterschiedlichen theoretischen Perspektiven auf das Thema zum Ausdruck kommen - auf. Er diskutiert die jeweiligen ontologischen und epistemologischen Standpunkte, die einen Einfluss auf die Entwicklung der unterschiedlichen Machtkonzepte haben, widmet sich ihren Stärken, Schwächen sowie verbindenden Elementen und geht der zentralen Frage nach, wie sie in der empirischen Forschung genutzt werden können. Er betrachtet unterschiedliche Konzeptionen von Macht als sich ergänzend nicht als exklusiv oder einzelne als falsch. Lukes bezieht sich auf Machtforscher*innen wie Max Weber, Robert Dahl, Nelson W. Polsby, Peter Bachrach oder Morton Baratz. Deren Ansätze entwickelt er weiter und integriert sie in ein mehrdimensionales Machtkonzept. Lukes eigener Schwerpunkt liegt auf der Konzeptualisierung der am wenigsten sichtbaren Formen der Macht: Über diese werde Macht am effektivsten ausgeübt (Lukes 2005: 1). Sein wesentlicher Anspruch an das Konzept ist, dass es Offenheit gegenüber empirischen Phänomenen gewährleistet, ohne sich auf eine a priori gesetzte moralische oder politische Perspektive zu beschränken (ebd.: 30). Damit eignet es sich insbesondere für explorativ angelegte Forschungsdesigns - wie dasjenige meiner Studie - die darauf abzielen, neue Erkenntnisse zu einem bisher wenig erforschten Phänomen zu gewinnen.

Das Kapitel ist wie folgt aufgebaut. Zunächst entwickele ich ein theoretisches Verständnis von Zugang zu Land in traditionellen Landrechtssystemen. 
Dafür arbeite ich rechtebasierte sowie relationale und strukturelle Mechanismen heraus, die für den Zugang zu Land in traditionellen Landrechtssystemen relevant sind. Diese Mechanismen bilden gleichzeitig die Kategorien für die Analyse meiner Daten. In einem zweiten Schritt stelle ich Steven Lukes' Machtkonzept dar. Aus diesem leite ich die Analysekategorien der sichtbaren, versteckten und unsichtbaren Macht ab. Im Anschluss daran erläutere ich, wie sich die beiden Konzepte kombinieren lassen. Schließlich gehe ich der Frage nach, wie sich die zuvor herausgearbeiteten theoretischen Kategorien mit Blick auf die Analyse der empirischen Daten operationalisieren lassen. In diesem Rahmen ordne ich jeder Kategorie Indikatoren und Beispiele zu, die Anhaltspunkte dafür liefern, wie sich die theoretisch hergeleiteten Kategorien in der Auswertung der empirischen Daten erfassen lassen. In diesem Rahmen diskutiere ich ebenfalls Herausforderungen und Schwächen, welche die theoretischen Kategorien hinsichtlich der empirischen Analyse mit sich bringen sowie den Umgang damit.

\subsection{Zugang als analytische Kategorie in Konflikten um Land}

Die Konzeptualisierung von Zugang zu Land als Fähigkeit und nicht als Recht, einen Nutzen aus Land ziehen zu können, ermöglicht es Zugang zu Land unter Berücksichtigung sozialer Verhältnisse $\mathrm{zu}$ analysieren und nicht allein über de jure Besitzverhältnisse. Gesetze und sich daraus ableitende Rechte egal ob schriftlich oder mündlich festgehalten, staatliche oder traditionelle können den vielschichtigen Zugang zu Land nicht hinreichend abbilden (Ribot/Peluso 2003: 156). Zugang als analytische Kategorie ermöglicht es hingegen, ein vollständigeres Bild der Mechanismen zu zeichnen, die beeinflussen, ob Akteure einen Nutzen aus Land ziehen können (vgl. Ribot 1998). Die einzelnen Mechanismen determinieren jedoch nicht den Zugang zu Land, sondern bedingen sich gegenseitig oder komplementieren sich. Gemeinsam bilden sie - in den Worten von Ribot und Peluso (2003: 154)- so genannte Machtbündel (bundles of power), auf die Personen und Institutionen zurückgreifen können. Diese Machtbündel sind akteurs- sowie kontextabhängig und die Machtbündel von Individuen, Gruppen oder Institutionen verweben sich zu Netzen der Macht (webs of power) (ebd.: 154). Demnach variiert, je nach Kontext, auch die Stellung der Akteure in diesen Netzen der Macht. Die Ausgestaltung des Zugangs zu Land ändert sich mit politisch-ökonomischem und institutionellem Wandel. Sich verändernde politisch-ökonomische und insti- 
tutionelle Rahmenbedingungen beeinflussen soziale Verhältnisse und wirken sich dadurch auf die Möglichkeiten von Gruppen und Individuen, Zugang zu Land zu kontrollieren oder (aufrecht) zu erhalten, aus (ebd.: 158). Auf welche Mechanismen Akteure zurückgreifen können, um Zugang zu erlangen oder aufrechtzuhalten, ist kontextabhängig. Daraus leite ich die Annahme ab, dass sich im Kontext von land grabbing die Zugangsmechanismen möglicherweise verändern.

In traditionellen Landrechtssystemen ermöglichen, neben rechtebasierten Mechanismen, relationale und strukturelle Mechanismen den Zugang zu Land (Berry 1992, 2018; Peters 2004). Rechtebasierte Zugangsmechanismen sind alle legalen sowie illegalen Mittel, die Zugang zu Land ermöglichen. Legale Mittel umfassen Gesetze, Gewohnheitsrechte oder individuelle Vereinbarungen, die gegenüber Dritten eingefordert werden müssen. Illegale Mittel können Diebstahl, Zwang oder Erpressung sein und dienen dazu, direkten Zugang zu Land zu erlangen, ohne dass Dritte diesen als legitim anerkennen. Rechtebasierte Mechanismen können, müssen jedoch nicht zwangsläufig mit der Fähigkeit verbunden sein, einen Nutzen aus Land zu ziehen. In vielen Fällen bedingen oder komplementieren relationale und strukturelle Mechanismen die rechtebasierten Mechanismen. Relationale und strukturelle Mechanismen gehen aus den kontextspezifischen Bedingungen hervor, unter denen Akteure nach Zugang zu Land streben. Sie beeinflussen die Möglichkeiten von Akteuren, Zugang zu Land zu erlangen. Zu relationalen und strukturellen Mechanismen zählen Möglichkeiten der Aushandlung sozialer und rechtlicher Ordnungen, in dessen Rahmen Akteure nach Zugang zu Land streben, das Engagement in Netzwerken sowie in sozialen Beziehungen, die Zugang zu Land ermöglichen. Darüber hinaus beeinflussen unterschiedliche Kategorien sozialer Zugehörigkeit, die mit strukturell begründeten Ungleichheitsverhältnissen einhergehen, die Möglichkeiten, Rechte an Land einzufordern und Zugang zu Land zu erlangen. Dazu zählen insbesondere Herkunft, Geschlecht und Alter (Berry 1989, 1993, 2009b, 2018; Juul/Lund 2002a; Lund 2011, 2013; Moore 1978; Sikor/Lund 2009b). Aber auch vorhandene Ressourcen wie Bildung oder Kapital - können rechtebasierten Zugang zu Land in traditionellen Landrechtssystemen stärken oder ermöglichen (Ribot/Peluso 2003: 167ff.). Rechtebasierte Zugangsmechnismen sind in vielfacher Weise mit relationalen und strukturellen Zugangsmechanismen verschränkt, bedingen sich gegenseitig und komplementieren einander. Rechte an Land sind beispielswese oftmals an soziale Zugehörigkeit geknüpft oder unterschiedliche soziale Zugehörigkeit kann mit strukturellen Ungleichheitsverhältnissen - beispiels- 
weise in Bezug auf Bildung oder Kapital - einhergehen. Eingeschränkter Zugang zu Land aufgrund sozialer Zugehörigkeit kann wiederum durch Beziehungen verbessert werden.

Machtverhältnisse können über die analytische Kategorie der Kontrolle in den Blick genommen werden. Zugang $\mathrm{zu}$ Land $\mathrm{zu}$ kontrollieren, bedeutet, überwachen, regulieren und lenken zu können, wer - auf welche Weise auch immer - einen Nutzen aus Land ziehen kann. Dies ist Ausdruck von Macht gegenüber denjenigen, die nicht über diese Fähigkeit verfügen und dementsprechend Zugang zu Land nur über Akteure erlangen und aufrechterhalten können, die ihn kontrollieren. Wer Kontrolle über den Zugang zu Land ausüben kann, ist eng an bestehende Rechte und damit an Vorstellungen von Eigentum und Besitz geknüpft. Gesetze, Normen und sich daraus ableitende Rechte sind jedoch nicht einmal etabliert und damit gesetzt, sondern Gegenstand sozialer Aushandlungsprozesse (Moore 1978). »Existing orders are endlessly vulnerable to being unmade, remade and transformed, and [...] even maintaining and reproducing themselves, staying as they are, should be seen as a process" (ebd.: 6). Rechte sind also immer in ihrem räumlichen und zeitlichen Kontext zu verstehen, der sich auf die Fähigkeit, Kontrolle über Zugang zu Land auszuüben und Zugang zu Land aufrechtzuerhalten, auswirken kann. »Property is not about things but about relationships between and among persons with regard to things " (Moore 1998: 33). Kontrolle und Aufrechterhaltung von Zugang zu Land gestalten die Beziehungen zwischen den unterschiedlichen Akteuren in Bezug auf die Aneignung und Nutzung von Land. Sowohl in Bezug auf die Kontrolle als auch auf die Aufrechterhaltung des Zugangs zu Land ist es daher für die Akteure zentral, kontinuierlich in relationale Zugangsmechanismen zu investieren (vgl. Berry 1993). Investitionen in relationale Mechanismen sind vor allem der Aufbau und die Pflege sozialer Beziehungen oder Netzwerke. Diese können unterschiedliche Formen annehmen, beispielsweise die anteilige Übertragung von Gewinnen - wie Ernteerträgen - an Akteure, die Kontrolle über den Zugang ausüben oder die Gewährung von Vorzügen - wie Arbeit - um sich Anerkennung zu sichern. Eine Person kann in einer Situation den Zugang zu Land kontrollieren, in einer anderen aber darauf angewiesen sein, ihn über eine andere Instanz zu erlangen. Die Kontrolle des Zugangs zu Land kann zwischen unterschiedlichen kooperierenden oder konkurrierenden Akteuren aufgeteilt sein. Akteure, die Zugangsmechanismen kontrollieren, nehmen in Konflikten dementsprechend eine beherrschende Stellung gegenüber denjenigen ein, die nicht über diese Fähigkeit verfügen (Ribot/Peluso 2003: 159). 
Im Folgenden gehe ich näher auf die einzelnen Mechanismen ein, die $\mathrm{Zu}$ gang zu Land in traditionellen Landrechtssystemen ermöglichen.

\section{Rechtebasierte Zugangsmechanismen}

Rechtebasierte Zugangsmechanismen umfassen legale sowie illegale Mittel, über die Zugang zu Land gesichert wird. Legaler Zugang beruht auf Rechten, die sich aus Gesetzen, Gewohnheit oder Vereinbarung ableiten. Diese ermöglichen es den Akteuren, Zugang zu Land gegenüber Dritten einzufordern. In staatlichem Recht erfolgt dies beispielsweise unter Berufung auf Grundbucheinträge, Besitzurkunden, Lizenzen oder Konzessionen. In traditionellen Landrechtssystemen dienen Gewohnheitsrecht oder individuell vereinbarte (mündliche oder schriftliche) Abkommen als Grundlage für die Einforderung von Zugang zu Land. Gewohnheitsrecht und individuelle Vereinbarungen beruhen in der Regel auf der Anerkennung sozialer Normen und Regeln, denen sich beide Seiten verpflichten. Für die Durchsetzung der auf diese Weise erlangten Zugangsansprüche bedarf es somit keinem Zwang. Je nach Kontext kann sich jedoch die Kontrolle über den Zugang zu Land ändern, so dass auch in traditionellen Landrechtssystemen teilweise Zwangsmaßnahmen zur Durchsetzung von Rechten zum Einsatz kommen. Dazu zählen beispielsweise bewaffneter Widerstand im Kampf gegen Enteignung oder Vertreibung von gewohnheitsrechtlichem Land. Diejenigen, die nicht die Möglichkeit haben, Rechte auf Land geltend zu machen oder sie auszuhandeln, sind darauf angewiesen, Zugang zu Land über diejenigen $\mathrm{zu}$ erhalten, die ihn kontrollieren. Im Gegenzug erbringen sie in der Regel eine Geld- oder Naturalleistung. In Kontexten, die durch Rechtspluralismus geprägt sind, geht rechtebezogener Zugang nicht selten mit Widersprüchen einher. Denn neben dem Gewohnheitsrecht - das seinerseits schon umkämpft sein kann besteht in der Regel auch die Möglichkeit, sich auf staatliches Recht zu beziehen. Die unterschiedlichen Rechtsgrundlagen überschneiden sich häufig in ihrer Reichweite, und unterschiedliche Institutionen können rechtebasierten Zugang zu Land gewährleisten. Rechtspluralismus eröffnet damit einigen Akteuren die Gelegenheit des forum shopping (vgl. von Benda-Beckmann 2002). Dieses bezeichnet die Möglichkeit, eigene Anliegen und Vorhaben in dem formal- oder gewohnheitsrechtlichen Raum zu verhandeln, der die eigenen Ziele besonders begünstigt. Jenseits der Rechtssphäre gewinnen daher hinsichtlich der Frage, wer staatliches Recht, Gewohnheitsrecht oder Konventio- 
nen wann und mit welcher Absicht nutzen kann, politisch-ökonomische und institutionelle Rahmenbedingungen an Bedeutung (Ribot/Peluso 2003: 157).

Illegaler Zugang lässt sich über die Abgrenzung zu bestehenden Rechten definieren, die sich aus Gesetzen, Gewohnheit oder Vereinbarungen ableiten. Die Unrechtmäßigkeit ist jedoch eine Frage der Perspektive oder des Verhältnisses einer Person zu bestimmten Rechtsgrundlagen (ebd.: 164). Dies kann am Beispiel einer Landnutzerin illustriert werden, die gewohnheitsrechtliche Ansprüche auf Land geltend macht, indem sie eine neuerrichtete physische Barriere, wie einen Zaun überwindet, um auf dem zuvor gemeinschaftlich genutzten Land Feuerholz zu sammeln. Nach staatlichem Recht handelt sie eventuell illegal oder kriminell, da sie formalisierte Eigentumsrechte verletzt hat. Laut Gewohnheitsrecht würde ihr die Nutzung des Landes möglicherweise jedoch zustehen. Illegaler Zugang bedeutet demnach mithilfe von Mitteln, die nicht durch staatliches Recht oder gewohnheitsrechtliche Regeln und Normen legitimiert sind, einen Nutzen aus Land zu ziehen. In vielen Fällen ist illegaler Zugang mit Zwang, Gewaltandrohung oder manifester Gewalt verbunden. Illegale Mittel dienen sowohl zur Kontrolle als auch zur Aufrechterhaltung des Zugangs zu Land. Beispiele für illegalen Zugang zu Land sind der Diebstahl von Ernteerträgen, die Sicherung einer Landfläche mit Waffengewalt durch nicht-staatliche Akteure oder die Kontrolle des Zugangs durch Vortäuschen falscher Tatsachen. Im Kontext von land grabbing stellen Unternehmensakteure häufig Sicherheitskräfte ein oder errichten physische Barrieren, um den Zugang zu (umstrittenem) Land zu kontrollieren, obwohl sie (noch) nicht über formalisierte Rechte an dem Land verfügen.

\section{Relationale und strukturelle Zugangsmechanismen}

Relationale und strukturelle Zugangsmechanismen bilden eine zusammenhängende übergeordnete Analysekategorie. Sie umfasst Zugangsmechnismen, die Akteure innerhalb sozialer Beziehungen im Rahmen struktureller Grenzen aushandeln. Strukturelle Rahmenbedingungen verstehe ich als nicht deterministisch, sondern kontext- und akteursabhängig. Durch Aushandlung können sie sich verändern. Relationale und strukturelle Mechanismen, die den Zugang zu Land in traditionellen Landrechtssystemen bestimmen, sind soziale Zugehörigkeit, Netzwerke und soziale Beziehungen.

Soziale Zugehörigkeit bildet die Grundlage für den Zugang zu Land und geht einher mit strukturellen Ungleichheitsverhältnissen. Insbesondere in traditionellen Landrechtssystemen, in denen nicht-staatliche Akteure - wie 
geistliche Führer, Dorfvorsteher oder traditionelle Autoritäten - den Zugang zu Land kontrollieren, ist dieser in der Regel selektiv an Merkmale sozialer Zugehörigkeit geknüpft. Seit präkolonialer Zeit ist Zugang zu Land in vielen Gesellschaften Afrikas mit der Zugehörigkeit zu oder der Akzeptanz durch eine bestimmte Abstammungsgruppe, Familie oder Gemeinschaft beziehungsweise der Loyalität gegenüber traditionellen Autoritäten verknüpft (Berry 2018: 6). Noch heute ist der Zugang zu Land für Außenstehende von der erfolgreichen Verhandlung ihrer Gruppenmitgliedschaft oder der Anerkennung traditioneller Landrechtsinstitutionen abhängig (Berry 1993: 104). Mit Beginn der Kolonialherrschaft haben Kolonialverwalter*innen über ihre Interpretation des Gewohnheitsrechts zudem den Grundsatz etabliert, dass traditionell verwaltetes Land kein individuelles Eigentum, sondern gemeinschaftlicher Besitz ist. Über diese Interpretation traditioneller Landrechte knüpften Kolonialverwalter*innen Zugang zu Land grundsätzlich an die Zugehörigkeit zu einer Gemeinschaft. Welche Gemeinschaft Zugang zu welchem Stück Land kontrolliert, ließen sie jedoch offen. Die Konsequenz war eine Konkurrenz zwischen unterschiedlichen Gruppen sowie die Neuverhandlung sozialer Zugehörigkeit, mit dem Ziel Zugang zu Land zu sichern (Berry 1993: 40; Crook 1986: 89). Mit der Zeit hat sich diese Kodifizierung traditioneller Landrechte in vielen Gesellschaften strukturell als rechtliche und soziologische Tatsache verankert. Die Sicherung von Zugang zu Land erfolgt bis heute unter Rückbezug auf die vermeintliche Zugehörigkeit zu einer bestimmten Gruppe. Mit dem Ziel Zugang zu Land zu kontrollieren, beziehen sich konkurrierende Akteure oder Gruppen auf im Widerspruch zueinander stehende normative Ordnungen oder führen unterschiedliche vermeintliche Präzedenzfälle aus der Vergangenheit an, um die Hoheit zu erlangen, Rechte an Land zu bestimmen (Lund 2011: 72). Die Zugehörigkeit $\mathrm{zu}$ einer Gemeinschaft, die erfolgreich Ansprüche auf Land geltend macht, ist in vielen Fällen die Voraussetzung, um Rechte an Land zu verteidigen, zieht jedoch nicht automatisch Rechte nach sich oder garantiert Individuen Zugang zu Land. Denn Konkurrenz um den Zugang zu Land besteht nicht nur zwischen unterschiedlichen Gruppen, sondern auch innerhalb von Gruppen. Nicht alle Mitglieder einer Gruppe genießen in dieser auch die gleiche Zugehörigkeit. Die Zugehörigkeit zu einer Gruppe leitet sich zunächst daraus ab, als Teil der Gemeinschaft akzeptiert zu sein und ein langfristiges Interesse daran zu haben, Mitglied der Gemeinschaft zu sein. Dazuzugehören bedeutet, Werte, Netzwerke und Gepflogenheiten zu teilen, andere anzuerkennen und somit Teil des Sozialgefüges zu sein (Anthias 2006: 21f.; 
Pfaff-Czarnecka 2011: 5). Beispiele für Kriterien der Integration in die Gemeinschaft sind eine gemeinsame Sprache, gemeinsame Essgewohnheiten, Musik, eine gemeinsame Religion oder das Feiern bestimmter Feste (ArhinSam 2019: 35f.). »Belonging [...] combines (1) perceptions and performance of commonality; (2) a sense of mutuality and more or less formalized modalities of collective allegiance, and (3) material and immaterial attachments that often result in a sense of entitlement." (Pfaff-Czarnecka 2011: 2, Hervorhebung im Original). Hinsichtlich der Möglichkeiten, Zugang zu Land zu erlangen, bezieht sich soziale Zugehörigkeit jedoch nicht nur auf die Zugehörigkeit zu der Gruppe, die das Land vermeintlich erobert, als erste besiedelt oder bestellt hat. Ebenfalls bedeutsam für den Zugang zu Land sind Kategorien wie Alter, Geschlecht, Klasse, Religion und Herkunft. Insbesondere Herkunft - verhandelt mittels Kategorien wie Indigenität oder Autochthonie sowie first comer/late comer - ist relevant (Lund 2011: 74). Das bedeutet, dass Zugang $\mathrm{zu}$ Land nicht für jedes Mitglied einer Abstammungsgruppe gleichberechtigt ausgestaltet ist und de facto nicht der gemeinschaftlichen Kontrolle unterliegt. Wanderfeldbäuerinnen und -bauern, mobile Tierhalter*innen, die arme Landbevölkerung, junge Menschen und Frauen gehören zu den benachteiligten Personengruppen. Machtvollen Akteuren hingegen kommt die flexible Aushandlung zugute, indem sie traditionelle Besitzrechte in Übereinstimmung mit engen Gruppeninteressen neu definieren (Amanor 2012: 735). Während Zugehörigkeit zu einer Gruppe potenziell Rechte an Land ermöglicht, kann Nicht-Zugehörigkeit dazu führen, dass die Möglichkeit, Ansprüche geltend zu machen, von vornherein verwehrt bleibt. Der Bezug auf Kategorien sozialer Zugehörigkeit zur Sicherung des Zugangs $\mathrm{zu}$ Land ist somit zentral. Strukturelle Ungleichheitsverhältnisse führen jedoch dazu, dass sein Nutzen sich zwischen unterschiedlichen Akteuren unterscheidet. Darüber hinaus hängt er auch davon ab, wieviel Einfluss zentralstaatliche Institutionen in der Anerkennung, Überwachung und Auflösung von Landrechtsansprüchen haben und entsprechend, wie groß der Verhandlungsspielraum lokaler Akteure ist (Lund 2011: 74).

Um Zugang zu Land zu erlangen und $\mathrm{zu}$ festigen ist nicht nur die soziale Zugehörigkeit von Akteuren zentral. Relevant ist ebenfalls das Engagement in Netzwerken. Netzwerke können ortsgebundene Gemeinschaften oder professionelle und politische Interessensverbände sein. Viele Menschen engagieren sich in diversen Netzwerken zugleich und nutzen diese mit wechselnden Prioritäten (Berry 1993: 133). Das ermöglicht es, sich eine Vielzahl unterschiedlicher Verhandlungswege und -möglichkeiten offen zu halten, über die Zugang 
zu Land gesichert werden kann (Berry 1993: 14f.). Um Zugang zu Land aushandeln zu können, ist die Möglichkeit, Einfluss auf Debatten und Entscheidungsprozesse nehmen zu können, zentral. Einfluss ist wiederum eng mit der Fähigkeit, Anhänger*innen bzw. Unterstützer*innen mobilisieren zu können verbunden, wofür Netzwerke vorteilhaft sind. Netzwerke müssen also nicht zwangsläufig mit einem unmittelbaren Nutzen verbunden sein, sondern sind eher auf langfristige Sicht von Bedeutung. Es kann beispielsweise von Vorteil sein, auf die exklusive Kontrolle von Zugang zu Land zugunsten der potenziell wichtigen Mitgliedschaft in einem Netzwerk zu verzichten, um verschiedene Aushandlungsmöglichkeiten in Bezug auf Zugang zu Land aufrechtzuerhalten (Berry 1993: 14). Akteure richten ihre Aufmerksamkeit mal mehr auf das eine, mal mehr auf das andere Netzwerk, wodurch die kollektive Handlungsfähigkeit im Rahmen von Netzwerken zu- oder abnehmen kann. Unabhängig davon bleiben Netzwerke aber soziale Räume, welche die Wiederaufnahme oder Neuverhandlung bestimmter Themen ermöglichen (Moore 1986: 275). Netzwerke sind deshalb gleichermaßen für mächtige wie für weniger mächtige Akteure relevant. Mächtige Akteure, die sich in Aushandlungsprozessen durchsetzen können und folglich relativ sicheren Zugang zu Land haben, benötigen Netzwerke, um - dank zahlreicher Anhänger*innen - ihren Einfluss aufrechterhalten zu können. Für weniger mächtige Akteure ist das Engagement in Netzwerken nötig, um erfolgreich soziale Zugehörigkeit aushandeln und überhaupt an Debatten teilhaben zu können. Die Aushandlung sozialer Zugehörigkeit und damit einhergehend die Verhandlungsfähigkeit hängen von einer Kombination aus zugeschriebenen und errungenen Qualifikationen ab, für die das Engagement in Netzwerken notwendig ist. Ebenso wie die Verhandelbarkeit durch soziale Differenzierung beeinflusst wird, ist auch die Möglichkeit sich in Netzwerken zu engagieren mit Kategorien sozialer Zugehörigkeit verknüpft (Amanor 1999: 44). Um in Netzwerke investieren $\mathrm{zu}$ können und als legitimes Mitglied anerkannt $\mathrm{zu}$ werden, ist materieller Wohlstand wichtig (Berry 1989: 42).

Der dritte und letzte Zugangsmechanismus, den ich unter relationale und strukturelle Zugangsmechanismen fasse, sind Beziehungen. Dies können soziale Beziehungen innerhalb der Gemeinschaft sein oder der Zugang zu Behörden (Ribot/Peluso 2003: 170), je nachdem, wer den Zugang zu Land kontrolliert. Soziale Beziehungen innerhalb der Gemeinschaft sind für viele Landnutzer*innen ein relevanter Zugangsmechanismus. Akteure ohne Zugang zu Land, können diesen über Beziehungen zu Akteuren, die Zugang zu Land kontrollieren, erlangen. Eine weitverbreitete Form von 
Zugang zu Land mittels sozialer Beziehungen ist das landwirtschaftliche Produktionssystem des sharecropping. Sharecropping ist eine Form der Pacht und bedeutet, dass Akteure, die Zugang zu Land kontrollieren, Pächter*innen (sharecropper) ihr Land für die Bewirtschaftung zur Verfügung stellen. Im Gegenzug erhalten sie einen vorher vereinbarten Anteil der erwirtschafteten Ernte, wodurch beide Parteien einen Nutzen aus dem Land ziehen können. Ebenso besteht auch die Möglichkeit, Zugang zu Land über eine klassische Pachtvereinbarung zu erhalten, in dessen Rahmen Akteuren Land gegen eine Geldleistung nutzen können. Beziehungen können darüber hinaus Zugang zu Land über die Vermittlung von Arbeitsplätzen ermöglichen. Der Nutzen dabei ist beidseitig. Im Sinne klientelistischer Beziehungen können Personen, die Arbeitsplätze kontrollieren, diese im Austausch diverser Gefälligkeiten vermitteln. Die Vermittlung von Arbeitsplätzen kann gleichzeitig auch als Investition in Netzwerke dienen, die zu einem späteren Zeitpunkt möglicherweise von Vorteil sind - auch um Zugang zu Land zu kontrollieren (Ribot/Peluso 2003: 167). Neben persönlichen Beziehungen innerhalb der Gemeinschaft sind, insbesondere mit Blick auf die Kontrolle über Zugang zu Land, auch Beziehungen zu unterschiedlichen Institutionen relevant. Die Beziehung zu diesen unterscheidet sich zwischen Akteuren, in Abhängigkeit wirtschaftlicher und sozialer Voraussetzungen. Beispielsweise sind Behörden oftmals weit entfernt und nicht jede* $r$ hat die entsprechenden Mittel - Geld, Transportmöglichkeiten, Zeit, Wissen - um sie aufzusuchen. Zudem konkurrieren Beamt*innen in Justiz- und Verwaltungsbehörden sowie traditionelle Autoritäten teilweise um sich überschneidende Zuständigkeitsbereiche. Akteure mit entsprechendem Wissen können dies im Sinne des forum shopping - der Möglichkeit, eigene Anliegen in dem formal- oder gewohnheitsrechtlichen Raum zu verhandeln, der die eigenen Ziele besonders begünstigt - zu ihrem eigenen Vorteil nutzen und sich über unterschiedliche Wege Zugang zu Land erschließen. Wissen - in diesem Fall Kenntnisse der eigenen Rechte oder Regeln der Landnutzung - kann auf ein hohes Bildungsniveau oder die Mitgliedschaft in Netzwerken, die Informationen zur Verfügung stellen, zurückzuführen sein. Akteure, denen entsprechendes Wissen fehlt, haben auch nicht die Möglichkeit des forum shopping. So kann eine wohlhabende Landnutzerin mit hohem Bildungsniveau, die aufgrund ihrer sozialen Zugehörigkeit traditionelle Rechte an Land erworben hat, diese dank ihres Wissens und ihrer finanziellen Mittel über Behörden formalisieren lassen, um sie zu stärken. Ein armer, wenig gebildeter Landnutzer hat diese Möglichkeit vielleicht nicht. Indessen unterhält er aufgrund seiner Loyalität 
aber möglicherweise eine gute Beziehung zu dem chief, der den Zugang zu Land kontrolliert. Diese Beziehung kann ebenfalls privilegierten Zugang zu Land ermöglichen, z.B. über die Vermittlung von Arbeit. Eine migrantische Landnutzerin hat vielleicht weder die eine, noch die andere Möglichkeit.

Bildung und Wissen sind auch über die Kenntnisse der eigenen Rechte hinaus relevant für den Zugang zu Land. Besonders deutlich wird dies an so genannten Expert*innen. Ein Expert"innenstatus - den Akteure über exklusive Informationen, spezialisiertes Wissen oder Hochschulabschlüsse mit ihren entsprechenden Titeln erlagen - kann Zugang zu Land in unterschiedlicher Weise beeinflussen. Die dem Expert*innentum zugeschriebene Kompetenz geht in vielen Fällen damit einher, Einfluss auf andere Akteure oder auf Diskurse, Normen und Regeln ausüben zu können. Dies kann wiederum Auswirkungen auf den Zugang zu Land haben. Beispielsweise rechtfertigen "wissenschaftliche« Narrative, die auf dem Wissen vermeintlicher Expert" innen beruhen, die großflächige Aneignung von Land durch Unternehmensakteure. Darüber hinaus kann exklusives Wissen in Form von Informationen dem eigenen Vorteil in Bezug auf Zugang zu Land dienen. Akteure, die $\mathrm{Zu}$ gang zu Land kontrollieren, können anderen Akteuren Informationen vorenthalten, um Widerstand zu vermeiden und die eigene Kontrolle des Zugangs aufrechtzuerhalten. Wissen kann ebenfalls ermöglichen, errungene Gewohnheitsrechte an Land über rechtliche Schritte aufrechtzuhalten und gegen den Verlust von Land zu kämpfen. Kenntnisse der eigenen Rechte und derjenigen anderer Akteure wirken sich auf den eigenen Handlungsspielraum aus. Nur wer die unterschiedlichen Möglichkeiten Zugang zu Land zu erlangen und zu kontrollieren, kennt, kann sie auch nutzen oder andere davon ausschließen. Soziale Zugehörigkeit, Netzwerke, soziale Beziehungen und strukturell begründete Ungleichheitsverhältnisse hängen zusammen und wirken sich auf die Kontrolle des Zugangs und den Zugang zu Land aus.

Soziale Zugehörigkeit, Netzwerke und soziale Beziehungen sind wandelbar und können sich - innerhalb struktureller Grenzen - im Laufe der Zeit über soziale Aushandlungsprozesse verändern, was sich wiederum auf den Zugang zu Land auswirken kann. Diese Verhandelbarkeit ist Gegenstand einer umfangreichen Literatur aus der Rechtsanthropologie und der historischen Sozialforschung. Studien, die sich in diesem Forschungsfeld verorten, verweisen darauf, dass traditionelle Landrechtssysteme in post-kolonialen Staaten Subsahara-Afrikas nicht statisch sind und auf fortbestehenden präkolonialen Regeln und Normen beruhen, sondern auf Basis der Interpretation von Tradition durch die Kolonialverwalter*innen entstanden sind (Chanock 
1985; Moore 1978; Ranger 1983). Es handelt sich also um neue Rechtssysteme die aus der kontextbezogenen Auslegung sozialer und rechtlicher Ordnungen heraus entstanden sind. Die Auslegung sozialer und rechtlicher Ordnungen in Bezug auf den jeweiligen relevanten Kontext ist nicht außergewöhnlich. Ganz im Gegenteil: die Ausübung jeglicher Rechtspraxis durch Jurist*innen besteht darin, rechtliche Grundlagen mit Bezug auf den jeweiligen Kontext zu interpretieren (Moore 1978: 1). Dies gilt auch für traditionelles Recht während der Kolonialzeit. Je nachdem welche Person an welchem Ort und zu welchem Zeitpunkt Auskunft darüber gab, war es Gegenstand unterschiedlicher Interpretation (Berry 2002: 645). Dies hat sich bis heute nicht geändert. Die Bedeutung und Auslegung bestimmter Konzepte des traditionellen Rechts bleiben Gegenstand sozialer Aushandlungsprozesse.

»Customs are constantly recreated and disputed. Men's custom is not always women's, elders' may not be juniors', and the tradition of the rich may differ from those of the poor - or be made to look as though they do « (Shipton/Coheen 1992: 308).

Verschiedene Individuen oder Gruppen schreiben $\mathrm{zu}$ unterschiedlichen Zeitpunkten und in unterschiedlichen Kontexten allgemein bekannten Konzepten, Begebenheiten oder Handlungen variierende Definitionen oder Bedeutungen $\mathrm{zu}$, um eigene Interessen durchzusetzen (Lund 2013; Peters 1984). Damit ist Verhandelbarkeit ein zentrales Merkmal von Gewohnheitsrechten (Berry 1993: 14). In Konflikten um Zugang $\mathrm{zu}$ Land ist Verhandelbarkeit als analytische Kategorie von besonderer Relevanz. Mit entsprechendem Geschick erweist sich die Mehrdeutigkeit des Gewohnheitsrechts für Konfliktakteure*innen als zentraler Vorteil, welcher der Verfolgung eigener Ziele dienen kann. Gleichzeitig können sich durch Verhandelbarkeit aber auch Widersprüche ergeben, die durch inkohärente oder inkompatible Ideen und Handlungen im Zusammenhang mit traditionellem Recht entstehen. Ein Beispiel für widersprüchliche Bedeutungszuschreibungen von Konzepten ist der Bezug auf Tradition selbst. Akteure können sich auf Tradition berufen, um gegenwärtig ausgeübte Vorgehensweisen zu legitimieren. Tradition kann als ein Vorbild dargestellt werden, dem es zu folgen gilt. Sie kann jedoch auch als ein veraltetes Model kritisiert werden, das es zu überwinden gilt (Peters 1984: 30). Widersprüche eröffnen potenziell neue Aushandlungsräume: Die Fähigkeit Zugang zu Land zu erlangen, steht in unmittelbarem Verhältnis $\mathrm{zu}$ der geschickten - auf die eigenen Ziele ausgerichteten - Interpretation von Gewohnheitsrecht. So ist die Bedeutung von Kategorien sozialer Zu- 
gehörigkeit für den Zugang zu Land oftmals diskursiv erzeugt. Diskursive Konstruktionen können inklusiver oder exklusiver Art sein. Sie können dazu dienen, den eigenen Zugang $\mathrm{zu}$ Land $\mathrm{zu}$ ermöglichen oder ihn anderen Individuen oder Gruppen zu verwehren. Diskursive Strategien, die dazu dienen Zugang zu Land neu zu verhandeln oder aufrechtzuerhalten, können konkurrierende Identitätsdiskurse, die Wiederbelebung, Erfindung oder das Nacherzählen von Geschichte sein (Lund 2013). Strategien der Exklusion zeichnen sich häufig durch Bestrebungen aus, vormals flexible Gruppengrenzen - bezüglich Familie, Abstammungslinie, Dorf, ethnisch definierten Gemeinschaften oder anderen gesellschaftlichen Einheiten - festzuschreiben. Damit geht eine engere Definition sozialer Zugehörigkeit einher. In Konflikten um Land ergibt sich daraus eine stärkere Abgrenzung der Rechteinhaber*innen gegenüber denjenigen, denen Rechte verwehrt bleiben (Peters 2004: 302ff.). Verhandelbarkeit kann sich somit positiv und negativ auf Zugang zu Land auswirken. Verhandelbarkeit dient nicht jeder Person oder Gruppe gleichermaßen, sondern sie steht in engem Zusammenhang mit bestehenden Sozialstrukturen (Amanor 1999: 44). Die erfolgreiche Auslegung von Tradition und damit der Regeln und Normen, die den eigenen Zugang $\mathrm{zu}$ Land bestimmen, ist an Kategorien sozialer Zugehörigkeit geknüpft. Bestehende Sozialstrukturen, so argumentiert Amanor (ebd.), zwingen die meisten Menschen dazu, der Interpretationen dessen, was machtvolle Akteure als gewohnheitsrechtlich definiert haben oder neu definieren, zu folgen. Wer sich an entsprechenden Debatten beteiligen und Diskurse beeinflussen kann, ist eine Frage von Macht und sozialer Differenzierung. In Bezug auf die relationalen Zugangsmechanismen soziale Zugehörigkeit, Beziehungen und Netzwerke ermöglicht die Berücksichtigung von Verhandelbarkeit eine differenziertere Analyse sozialer Verhältnisse.

Wer auf welche Zugangsmechanismen zurückgreifen und darüber $\mathrm{Zu}$ gang zu Land erlangen, aufrechterhalten oder kontrollieren kann, hängt von sozialer Differenzierung und Machtverhältnissen ab. Gleichwohl die Kategorien der relationalen und strukturellen Zugangsmechanismen soziale Differenzierung berücksichtigen, fehlt ihnen ein erklärendes Moment, das Aufschluss darüber gibt, woher sich die Fähigkeit einzelner Akteure, Zugang $\mathrm{zu}$ Land $\mathrm{zu}$ kontrollieren - oder mit anderen Worten ihre Macht in Konflikten um Land -ableitet. Mit dem Ziel dies zu analysieren, nutze ich ein Machtkonzept, das Akteurshandeln in Beziehung zu strukturellen Faktoren wie politisch-ökonomischen und institutionellen Rahmenbedingungen, 
gesellschaftlich vorherrschenden Normen sowie internalisierten Denk- und Handlungsmustern setzt.

\subsection{Macht als analytische Kategorie in Konflikten um Zugang zu Land}

Mit dem Ziel analysieren zu können, über welche Handlungsstrategien es traditionellen Autoritäten gelingt, Zugang zu Land zu kontrollieren und wie sich diese Fähigkeit erklären lässt, verknüpfe ich die unterschiedlichen analytischen Kategorien des Zugangs mit dem von Steven Lukes (2005) entwickelten Konzept zur Analyse von Machtverhältnissen. Lukes unterscheidet zwischen drei Auffassungen von Macht, die er als eindimensionale, zweidimensionale und dreidimensionale Perspektive bezeichnet (ebd.: 15). Andere Autor*innen, die an Lukes' Konzept anschließen, bezeichnen diese drei Perspektiven als sichtbare (visible), versteckte (hidden) und unsichtbare (invisible) Macht (vgl. Gaventa 2006; VeneKlasen/Miller 2002). Diese Bezeichnungen übernehme ich im Folgenden. Sichtbare, versteckte und unsichtbare Macht schließen sich nicht gegenseitig aus, sondern bauen aufeinander auf und ergänzen sich.

\section{Sichtbare Macht}

Das Verständnis von Macht, das ich im Folgenden als sichtbare Macht bezeichne, geht insbesondere auf Arbeiten so genannter pluralistischer Theoretiker wie Robert Dahl (1957, 1961) oder Nelson Polsby (1960), zurück. Sie fassen Macht als formelle Entscheidungschancen, die von jeder Position innerhalb der Sozialstruktur gleichermaßen zugänglich, also pluralistisch verteilt sind (Offe 1977: 8). Aus dieser Perspektive ist Macht nicht in den Händen einer kleinen Elite gebündelt, sondern verteilt sich auf verschiedene gesellschaftliche Gruppen und Individuen (Lukes 2005: 5). Das erklärt sich darüber, dass Macht immer an Gegenstände geknüpft ist, die vergänglich oder andauernd sind und zu kurz- oder auch längerfristigen Koalitionen zwischen unterschiedlichen Akteuren führen können, jedoch nicht zeitlos oder unveränderlich sind (Polsby 1960: 478f.), Theoretisch schließt dieses Machtverständnis an den repressiven Machtbegriff nach Max Weber (1972 [1922]) an. Weber definiert Macht als "jede Chance innerhalb einer sozialen Beziehung den eigenen Willen auch gegen Widerstreben durchzusetzen« (1972 [1922]: 28). In Anlehnung daran beschreibt Dahl (1961) Macht als die Fähigkeit einer Person, 
eine andere Person dazu zu bringen etwas zu tun, das sie sonst nicht tun würde (Dahl 1957: 202f.). Dahl spezifiziert darüber hinaus, dass Macht den erfolgreichen Versuch einer Person, eine andere Person dazu zu bringen etwas zu tun, das sie sonst nicht tun würde, bedeutet (ebd.: 204). Diese Spezifizierung verweist auf einen zentralen Punkt in der Debatte um Macht, nämlich auf die Unterscheidung zwischen möglicher und tatsächlicher Macht, zwischen dem Machthabe und Machtausübung (Lukes 2005: 17). Das Konzept der sichtbaren Macht konzentriert sich auf letztere. Eine Untersuchung von Machtverhältnissen aus dieser Perspektive beschränkt sich auf die Untersuchung von Akteurshandeln. Ein beliebter Untersuchungsgegenstand, der dies ermöglicht, sind Entscheidungsfindungsprozesse zu Themen, über die es einen beobachtbaren Interessenskonflikt gibt. Macht bedeutet in diesem $\mathrm{Zu}$ sammenhang, in der Lage zu sein, Entscheidungen zu umstrittenen Themen gegen Widerstreben durchzusetzen (ebd.: 19). Eine solche Analyse von Machtverhältnissen setzt die Annahme voraus, dass Interessen bewusst sind und kommuniziert werden. In Anlehnung an Hartwig Schuck (2014: 304) bezeichne ich diese Interessen als manifeste Interessen. Damit grenze ich mich bewusst von Lukes missverständlicher Bezeichnung der subjektiven Interessen ab. Manifeste Interessen sind somit Bestrebungen oder Präferenzen, die Akteure formulieren und/oder die sich aus ihren Handlungen ablesen lassen, "gleichviel auf welchen ursprünglichen Motiven und Ursachen sie beruhen mögen« (ebd.). Über das Handeln gemäß (widersprüchlicher) Interessen und ihrer Kommunikation zeigt sich ein für Forschende beobachtbarer Konflikt, in dem Machtverhältnisse in Form von Entscheidungen zu strittigen Themen oder umkämpften Gegenständen deutlich werden.

\section{Versteckte Macht}

Der Fokus auf der tatsächlichen Ausübung von Macht anhand der Durchsetzung von Entscheidungen ist Gegenstand der Kritik des Konzepts der sichtbaren Macht (Bachrach/Baratz 1962: 948).

Ausgehend von dieser Kritik erweitern Peter Bachrach und Morton S. Baratz das Konzept der sichtbaren Macht um ein »zweites Gesicht« (1962) und damit um ein Verständnis von Macht, das ich in Anlehnung an John Gaventa (2006) sowie Lisa VeneKlasen und Valerie Miller (2002) als versteckte Macht bezeichne. Denn Macht, so argumentieren Bachrach und Baratz, bedeutet nicht ausschließlich, Entscheidungen gegen Widerstreben treffen $\mathrm{zu}$ können, sondern auch, mögliche alternative Entscheidungen, die den eige- 
nen Interessen entgegenstehen, von vornherein auszuschließen. In diesem Sinne umfasst Macht ebenfalls die Fähigkeit, politische und soziale Werte oder institutionelle Verfahrensweisen zu begünstigen, die dazu beitragen, dass nur solche Themen auf die politische Agenda oder in die öffentliche Diskussion gelangen, die nicht im Widerspruch zu den eigenen Interessen stehen bzw. diese nicht gefährden. Dadurch bekommen Gegner*innen für wichtige Anliegen keine politische Bühne und es wird ihnen so erschwert, öffentliche Aufmerksamkeit für umstrittene Themen zu gewinnen (ebd.: 948). Die Ausübung versteckter Macht umfasst darüber hinaus, bestimmen oder beeinflussen zu können, wer auf welche Weise an Entscheidungsprozessen teilnehmen kann und wer nicht. Die Analyse versteckter Machtverhältnisse untersucht, welche Themen gar nicht erst zur Diskussion stehen und daher auch nicht zum Gegenstand von Entscheidungen werden. Denn »[a]n dem Punkt eines Machtprozesses, an dem [die Analyse der sichtbaren Macht] ihre Beobachtungen aufnimmt, ist sozusagen das Wichtigste schon passiert: die Aussonderung von Alternativen, die dann auf der Ebene manifesten Entscheidungshandelns und beobachtbaren Widerstrebens keine Rolle mehr spielen« (Offe 1977: 14f.). Im Zentrum der Analyse versteckter Macht stehen damit die - wie Bachrach und Baratz es ausdrücken - »Nicht-Entscheidungen« (nondecisions) (1962: 949), die als Hinweis auf die Ausübung versteckter Macht gelten. Mit dem Begriff Nicht-Entscheidungen ist gemeint, dass machtvolle Akteure Widersprüche in den Einstellungen von Akteuren und damit potenzielle Konflikte - die Forschende zu identifizieren haben - strategisch aus Entscheidungsprozessen heraushalten und damit verhindern, dass sie institutionell ausgehandelt werden und Eingang in öffentliche Debatten finden. NichtEntscheidungen sind damit aktive Entscheidungen, die Aushandlung konfliktiver Themen zu vermeiden (Bradshaw 1976: 124). Nicht-Entscheidungen sind somit empirisch beobachtbare Entscheidungen, die dazu führen, dass latente oder manifeste Herausforderungen etablierter Werte oder Interessen von Entscheidungsträger*innen verdrängt oder ausgebremst werden. Sie sind ein Mittel, um Forderungen nach Veränderung - z.B. in Bezug auf die Verteilung von Privilegien - im Keim zu ersticken oder verdeckt zu halten, bevor sie ausgesprochen werden oder relevante Entscheidungsräume erreichen können (Bachrach/Baratz 1970: 44). In Hinblick darauf ist ein weiterer zentraler Punkt für die Analyse versteckter Macht die Berücksichtigung der vorherrschenden Werte, Überzeugungen und institutionellen Verfahrensweisen - kurz der "Spielregeln« - die sich zugunsten bestimmter Akteure und auf Kosten anderer auswirken (Bachrach/Baratz 1962: 950). Denn alle Formen 
politischer Organisation tendierten dazu, bestimmte Konflikte zu nutzen und andere auszublenden. Diese Tendenz bezeichnet Elmer Eric Schattschneider als mobilization of bias: "Some issues are organized into politics while others are organized out« (1960: 71).

Im Gegensatz zu dem Konzept der sichtbaren Macht beinhaltet dasjenige der versteckten Macht ein breiteres Verständnis von Interessen. Im Konzept der sichtbaren Macht sind Interessen die politischen Präferenzen, die sich in Entscheidungsprozessen - an denen vermeintlich jede* $r$ teilhaben kann - abzeichnen. Das Konzept der versteckten Macht berücksichtigt darüber hinaus die Interessen derjenigen, die teilweise oder gänzlich von Entscheidungsprozessen ausgeschlossen sind. Forschende können diese Interessen anhand offener oder verdeckter Missstände, auf die Akteure hinweisen, identifizieren. Offene Missstände sind solche, die Akteure öffentlich äußern und die bereits Diskussionsgegenstand der politischen Agenda sind. Verdeckte Missstände sind solche, die Akteure benennen, die es jedoch noch nicht in die öffentliche Aufmerksamkeit oder gar in politische Entscheidungsräumen geschafft haben (Bachrach/Baratz 1970: 49). Trotz dieses breiteren Verständnisses von Interessen folgen Bachrach und Baratz jedoch weiterhin der Annahme, Interessen würden bewusst als Bedürfnisse oder Präferenzen formuliert und seien beobachtbar (Lukes 2005: 24).

An diesem Punkt setzt Lukes mit seiner Kritik an, die drei zentrale Punkte umfasst. Er kritisiert erstens, dass das Konzept der versteckten Macht mit seinem Fokus auf beobachtbares (Konflikt-)Handeln ebenso wie das der sichtbaren Macht die Ausübung von Macht am Widerstreben unterlegener Akteure ausmache und damit Machtverhältnisse übersähe, die in Inaktivität unterlegener Akteure resultieren. Dies sei problematisch, da die Ausrichtung eines Systems nicht allein über die Handlungen einzelner Individuen oder Gruppen aufrechtgehalten wird, sondern insbesondere über sozial und kulturell strukturiertes Verhalten oder institutionelle Verfahrensweisen. Inaktivität könne diese Strukturen zusätzlich festigen. Zweitens kritisiert Lukes die Annahme, dass für die Analyse von Machtverhältnissen ein beobachtbarer Konflikt vorhanden sein muss. Denn Macht werde nicht nur ausgeübt, wenn eine Person eine andere Person dazu bringt, etwas zu tun, das sie ansonsten nicht tun würde. Macht auszuüben bedeute auch, die Interessen und Bedürfnisse einer anderen Person so zu prägen, $\mathrm{zu}$ beeinflussen und letztendlich $\mathrm{zu}$ bestimmen, dass Missstände und die ihnen zugrundeliegenden Widersprüche erst gar nicht als solche wahrgenommen und erkannt würden. Alternativen zum Status quo würden in diesem Fall nicht in Betracht gezogen, da dieser 
als selbstverständlich, unveränderbar oder vorteilhaft wahrgenommen würde (ebd.: 28). Auf diese Weise wirkten unterlegene Personen willig an ihrer Unterdrückung mit. Macht zeige sich damit nicht nur in manifesten Konflikten, sondern auch in ihrer Verhinderung oder Unterdrückung (ebd.: 27). Lukes dritter Kritikpunkt betrifft die implizite Annahme, auf der das Konzept der versteckten Macht beruht, dass Nicht-Entscheidungen nur dann vorliegen, wenn Missstände und damit eindeutig gegensätzliche Interessen identifiziert werden können, die nicht in Form von Themen Eingang in Entscheidungsräume finden. Das müsse im Umkehrschluss bedeuten, dass die vermeintliche Abwesenheit von Missständen bedeute, dass es auch keine Interessen gibt, die durch die Ausübung von Macht verletzt werden könnten (ebd.: 28).

\section{Unsichtbare Macht}

Anknüpfend an seine Kritik entwickelt Lukes das Konzept der unsichtbaren Macht, das über beobachtbares (Konflikt-)Handeln hinausgeht und damit die Konzepte der sichtbaren und versteckten Macht ergänzen soll. Eine Analyse der unsichtbaren Macht ist dazu bestimmt, Faktoren zu ermitteln, die Akteure daran hindern, Missstände und die ihnen zugrundeliegenden widersprüchlichen Interessen als solche zu erkennen. Damit bleiben auch die Mechanismen, die bewirken, dass potenzielle Themen gar nicht erst auf die Agenda gelangen, im Verborgenen. Um unsichtbare Macht in den Blick nehmen zu können, ist es notwendig, gleichermaßen gesellschaftliche Kräfteverhältnisse, institutionelle Verfahrensweisen und individuelles Handeln in Form von Entscheidungen zu berücksichtigen. Dies kann im Kontext eines manifesten, beobachtbaren Konflikts oder in seiner Abwesenheit geschehen; denn die Ausübung versteckter Macht kann einen manifesten Konflikt ggf. verhindern. Implizit bleibt dann ein latenter Konflikt bestehen. Relevant für die Analyse unsichtbarer Macht ist das Vorhandensein eines latenten Konflikts. Dieser äußert sich anhand eines Widerspruchs zwischen den Interessen der Machtausübenden und den, wie Lukes sie bezeichnet, »wirklichen« Interessen (real interests) derjenigen, die aufgrund von Machtverhältnissen ausgegrenzt werden. Letztere müssen sich laut Lukes - ebenfalls auf Grund existierender Strukturen und Machtverhältnisse - ihrer Interessen nicht bewusst sein oder sie äußern (ebd.). Dies lässt vermuten, es handele sich um Interessen, deren Identifizierung nicht durch die betroffenen Personen selbst erfolgen könne, jedoch sehr wohl durch Außenstehende. In der Rezeption von Lukes Konzept der unsichtbaren Macht interpretieren einige Autor*innen 
daher »wirkliche« als »objektive« Interessen im marxistischen Sinn (vgl. u.a. Benton 1981; Dowding 2006). Im klassischen Marxismus sind Interessen gleichbedeutend mit kollektiven Interessen oder Klasseninteressen. Konflikte sind aus dieser Perspektive auf widersprüchliche Interessen zurückzuführen, die sich aus der ungleichen Verteilung von Ressourcen und Macht ergeben (Turner 1975). Interessen werden in diesem Zusammenhang als »objektiv« gegeben angenommen. Entsprechend können Interessen Akteuren von außen zugeschrieben werden, ohne dass sie sich ihrer selbst bewusst sein müssen. Die Annahme solcher »objektiver « Interessen wurde vielfach als teleologisch, essentialistisch und reduktionistisch kritisiert (Sibeon 2004). Demgegenüber vertritt Lukes die Ansicht, Personen oder Gruppen, deren »wirkliche« Interessen durch die Ausübung von Macht verletzt werden, müssten eben diese Interessen selbst identifizierten können, insofern sie nicht durch Machtverhältnisse daran gehindert würden (Lukes 2005: 37). Sein Verständnis von »wirklichen« Interessen unterscheidet sich damit vom marxistischen Begriff der »objektiven« Interessen. Allerdings argumentiert Lukes an späterer Stelle, um unsichtbare Macht überhaupt erkennen $\mathrm{zu}$ können, bedürfe es eines normativen Urteils von außen (ebd.: 146). Ted Benton (1981) bezeichnet dies als paradox of emancipation. Damit bezieht er sich auf die Frage, wie eine Selbst-Emanzipation (oder bei Lukes die Identifizierung der wahren Interessen durch die Akteure selbst) erfolgen kann, wenn angenommen wird, dass die soziale Ordnung, in der die Akteure leben, ihr Bewusstsein beeinflusst bzw. systematisch manipuliert (ebd.: 162). Lukes selbst liefert keine Lösung für dieses Problem. Mit Blick auf die Analyse bleibt die Identifizierung der »wirklichen« Interessen damit schwierig.

Einen möglichen Lösungsansatz für diese Problematik bietet Schuck (2014) an. Er spricht sich für den Begriff der »ethischen Interessen« aus. Unter ethischen Interessen versteht er solche, die Akteuren »ein gutes Leben ermöglichen (würden)« (ebd.: 305). Diese ergeben sich, so argumentiert Schuck, aus »einer adäquaten kritischen Analyse der herrschenden Verhältnisse« (ebd.: 306) und aus "Annahmen über die menschliche Natur und das gute Leben« (ebd.: 306). Damit ist die Ermittlung von Interessen weiterhin abhängig von »ethischen Prämissen und Hypothesen bzw. - im historischen Rückblick - kontrafaktischen Entwicklungsverläufen« (ebd.: 306), auf die ich später im Abschnitt zur Operationalisierung der theoretischen Kategorien näher eingehe. Die Behauptung, dass entgegen der ethischen Interessen einer Person gehandelt wurde, impliziert die Annahme, dass die Handlung dem guten Leben dieser Person schadet. 
Tabelle 1: Übersicht über die drei Dimensionen der Macht

\section{Sichtbare Macht}

$\begin{array}{lll}\text { Betrachtung von... } \quad . & \text { Handeln } \\ & \text { Entscheidungsfindung } \\ . & \text { (zentralen) Themen } \\ . & \text { beobachtbaren (manifesten) Konflikten } \\ \text { manifesten Interessen, die Akteure formulieren und/oder die } & \text { sich aus ihren Handlungen ablesen lassen }\end{array}$

Versteckte Macht

Betrachtung von... . Entscheidungsfindung und »Nicht-Entscheidungen«

- Themen und potenziellen Themen

- beobachtbaren (manifesten und versteckten) Konflikten

- (manifesten) Interessen in Form politischer Präferenzen oder Missstände

\section{Unsichtbare Macht}

Betrachtung von... $\quad$. Entscheidungsfindung und Kontrolle über Agenda-Setting (nicht notwendigerweise anhand von Entscheidungen)

- Themen und potenzielle Themen

- beobachtbaren (manifesten und versteckten) sowie latenten Konflikten

manifesten und ethischen Interessen

Quelle: Eigene Darstellung in Anlehnung an Lukes (2005: 29)

\subsection{Zwischenfazit}

Um $\mathrm{zu}$ analysieren, in welchem Zusammenhang das Handeln traditioneller Autoritäten mit Konflikten um Zugang zu Land stehen, erweist sich die Kombination theoretischer Kategorien von Zugangsmechanismen mit einem dif- 
ferenzierten mehrdimensionalen, auf die empirische Analyse ausgerichteten Verständnis von Macht aus unterschiedlichen Gründen als sinnvoll.

Zugang als analytische Kategorie ermöglicht es, nicht nur bestehende Rechte an Land in der Untersuchung zu berücksichtigen, sondern den analytischen Blick zu weiten und alle Mechanismen $\mathrm{zu}$ erfassen, die Akteure befähigen, Zugang zu Land zur erlangen, also- auf welche Weise auch immer - einen Nutzen aus dem Land zu ziehen. Diese Mechanismen können in Konflikten um den Zugang zu Land Gegenstand von Aushandlungsprozessen und damit umstritten sein. Zugangsmechanismen sind strukturierende Elemente in Konflikten um Land und stellen als solche wichtige Ansatzpunkte für die Analyse von Konflikten um Land dar. In vielen Fällen lässt sich entlang von Zugangsmechanismen und ihrer Veränderung im Kontext von Transformationsprozessen nachvollziehen, worum es in den Konflikten geht. Darüber hinaus erlaubt es die Analyse von Zugangsmechanismen, eine Vielzahl möglicher Widersprüche zu erfassen, die auf die Heterogenität der Konfliktakteure und ihres Zugangs zu Land zurückgehen und Konflikten zugrunde liegen.

Jenseits der Analyse, über welche Mechanismen unterschiedliche Personen Zugang zu Land erlangen, liegt das zentrale Interesse meiner Studie auf der Kontrolle des Zugangs und der Frage, wie Kontrolle erlangt, erweitert und aufrechterhalten wird. Die Kontrolle des Zugangs zu Land ist in diesem $\mathrm{Zu}$ sammenhang als Ausdruck von Macht zu verstehen.

In der Vergabe und Kontrolle von traditionell verwaltetem Land sind traditionelle Autoritäten zentrale Akteure. Der analytische Fokus liegt daher auf der Macht traditioneller Autoritäten und den damit zusammenhängenden Möglichkeiten, Zugang zu Land zu kontrollieren. Da ich Macht als relational verstehe, ist die Macht traditioneller Autoritäten in Konflikten um Zugang zu Land immer in Bezug zu anderen Konfliktakteuren zu sehen. Für die Analyse bedeutet das, die unterschiedlichen Akteure in ihrem Verhältnis zueinander zu sehen und neben traditionellen Autoritäten ebenso alle anderen relevanten Akteure zu berücksichtigen.

Die Verknüpfung der unterschiedlichen Zugangsmechanismen mit einem differenzierten Verständnis von Machtverhältnissen bedeutet für die Analyse der empirischen Daten Folgendes: Zunächst muss sie untersuchen, inwiefern unterschiedliche rechtebasierte, strukturelle sowie relationale Zugangsmechanismen in den erforschten Konflikten relevant sind und zwischen welchen Akteuren sie auf welche Weise ausgehandelt werden. Darauf aufbauend ist das Ziel der Analyse, herauszuarbeiten, inwiefern sich unterschiedliche 
Machtformen in Konflikten auf die Fähigkeit Zugang zu Land zu kontrollieren, auswirken.

\subsection{Operationalisierung der theoretischen Kategorien}

Die zuvor erarbeiteten zentralen Analysekategorien für meiner Studie sind rechtebasierte, strukturelle und relationale Zugangsmechanismen sowie sichtbare, versteckte und unsichtbare Macht. Für die empirische Analyse erarbeite ich Indikatoren, anhand derer ich erkenne, dass es sich bei einem in der Forschung beobachteten Phänomen um einen Zugangsmechanismus oder eine Form von Macht handelt.

\section{Operationalisierung der Zugangsmechanismen}

Die Identifizierung der im Konflikt umkämpften Zugangsmechanismen zu Land dient der Strukturierung der Analyse und soll den Blick für mögliche Konfliktgegenstände schärfen. Welche Zugangsmechanismen in den untersuchten Konflikten um Land von Bedeutung sind, erfasse ich analytisch über die Veränderung von Zugangsbedingungen. Hinweise auf Veränderungen von Zugangsbedingungen ermittle ich über die Fragen, wie und auf welcher Grundlage Akteure vor der Aneignung des Lands durch das Unternehmen Zugang zu diesem erlangt haben und mit welchen Konsequenzen sich dies im Kontext von land grabbing verändert hat. Im Folgenden entwickle ich Indikatoren, anhand derer ich rechtebasierte, relationale und strukturelle Zugangsmechanismen in der Analyse erkennen kann.

Rechtebasierte Zugangsmechanismen sind legale Mittel, die Zugang $\mathrm{zu}$ Land über gesellschaftlich anerkannte Rechte ermöglichen, sowie illegale Mittel, die in Abgrenzung zu legalen Mitteln existieren, also nicht auf gesellschaftlicher Akzeptanz beruhen und Zugang zu Land über nichtinstitutionelle Wege ermöglichen. In traditionellen, durch rechtspluralistische Strukturen geprägten Landrechtssystemen finden gesellschaftlich anerkannte Rechte in den folgenden Formen Ausdruck: in behördlichen Besitzurkunden, schriftlich oder mündlich festgehaltenen Pachtverträgen, in Landnutzungsrechten, die traditionelle Institutionen oder individuelle Landrechteinhaber*innen vergebenen oder in sharecropping-Vereinbarungen. Auch Entschädigungszahlungen für den Verlust von Land zählen zu den rechtebasierten Zugangsmechanismen, da die Voraussetzung für ihren 
Erhalt zuvor bestehende Rechte an Land sind. Legale Mittel können den eigenen Zugang zu Land gewährleisten oder ermöglichen, Zugang zu Land an Dritte zu übertragen. Gegenleistungen für die Übertragung von Rechten in Form von Naturalien oder Pachtzahlungen führen dazu, dass beide Seiten einen Nutzen aus dem Land ziehen. Illegale Mittel umfassen die Androhung oder Anwendung von Gewalt, Diebstahl oder das Vortäuschen falscher Tatsachen. Letzteres bedeutet, dass Akteure Zugang zu Land erlangen, indem sie - obgleich dies nicht der Fall ist - behaupten, über eine Besitzurkunde oder traditionelle Rechte an Land zu verfügen. Insbesondere in Bezug auf traditionelle Rechte an Land, für die es keinen handfesten Beweis in Form einer vorzeigbaren Besitzurkunde gibt, kann es empirisch schwierig sein, eindeutig zwischen legalen oder illegalen Mitteln zu unterscheiden. Denn die Behauptungen unterschiedlicher Akteure können sich diesbezüglich widersprechen. Indikator für rechtebasierte Zugangsmechanismen ist der Einsatz legaler oder illegaler Mittel, um Zugang zu Land einzufordern. Beispiele für Indikatoren rechtebasierter Zugangsmechanismen sind, dass Akteure sich auf seit Generationen bestehende traditionelle Landrechte berufen oder Besitzurkunde vorweisen, um Zugang zu Land einzufordern. Ein weiteres Beispiel ist die Androhung von Waffengewalt, um sich Zugang zu Land zu verschaffen.

Relationale und strukturelle Zugangsmechanismen komplementieren die rechtebasierten Zugangsmechanismen, insbesondere in traditionellen Landrechtssystemen. Relevante relationale und strukturelle Zugangsmechanismen in traditionellen Landrechtssystemen sind soziale Zugehörigkeit, Netzwerke und soziale Beziehungen. Diese Zugangsmechanismen sind, im Rahmen struktureller Grenzen, verhandelbar und stehen in einem wechselseitig konstitutiven Verhältnis zu rechtebasierte Zugangsmechanismen. Soziale und institutionelle Rahmenbedingungen, die Zugang zu Land ermöglichen, sind Gegenstand sozialer Aushandlungsprozesse. Sie sind nicht einmal geschaffen und damit unveränderbar, sondern stehen in einem wechselseitigen Verhältnis zu dem Handeln von Akteuren. Soziale und institutionelle Rahmenbedingungen beeinflussen das Handeln von Akteuren, das seinerseits wiederum auf die Ausgestaltung der Bedingungen zurückwirkt. Akteure verleihen sozialen und institutionellen Rahmenbedingungen Legitimität und tragen $\mathrm{zu}$ ihrem Fortbestand bei, indem sie sie anerkennen. Oder sie verändern sie, indem sie sie umdeuten. Verhandelbarkeit als analytische Kategorie muss daher in Bezug auf alle Zugangsmechanismen mitberücksichtigt werden. Indikatoren für die Verhandelbarkeit von 
Zugangsmechanismen bestehen darin, dass Akteure existierende soziale und institutionelle Rahmenbedingungen infrage stellen oder neu auslegen, um Zugang zu Land zu erlangen oder anderen Akteuren den Zugang zu verwehren. Beispiele hierfür sind die Umdeutung oder Neuinterpretation traditioneller Landrechte zu Gunsten bestimmter Akteure sowie Bestrebungen von Akteuren, die eigene Zugehörigkeit oder diejenige anderer, neu auszulegen, mit dem Ziel, Zugang zu Land zu erlangen oder zu beschränken.

Soziale Zugehörigkeit, die mit strukturell begründeten Ungleichheitsverhältnissen einhergeht, beeinflusst die Möglichkeiten, Zugang zu Land - insbesondere rechtebasierten Zugang - zu erlangen. Indigene Landnutzer*innen - also diejenigen, die vorgeben schon immer in der Gegend gelebt $\mathrm{zu}$ haben oder die Ersten gewesen zu sein, die das Land bestellt haben - verfügen in der Regel über permanente Rechte an Land. Je nach Kontext können diese Rechte für Mitglieder der Gemeinschaft übertragbar sein. Teilweise beschränkt sich dieses Recht auf Männer. Zugezogene Landnutzer*innen aus anderen Staaten oder Gegenden erhalten in der Regel nur temporäre Rechte. Für indigene Landnutzer*innen bleiben Rechte an Land auch bestehen, wenn sie es eine Weile brach liegen lassen. Je nach sozialer Zugehörigkeit sind für manche Landnutzer*innen Rechte zudem mit Pflichten verbunden. Der Erhalt von Weide- oder Anbaurechten kann mit der Abgabe landwirtschaftlicher Erzeugnisse einhergehen (Shipton/Goheen 1992: 311). Indikator für soziale Zugehörigkeit als relevanter Mechanismus für Zugang zu Land ist die Einforderung, Legitimierung oder Beschränkung von Zugang zu Land über Kategorien sozialer Zugehörigkeit wie Indigenität, first comer/late comer, Herkunft, Geschlecht oder Alter. Auch Wissen spielt eine wichtige Rolle in Bezug auf Zugang zu Land. Wissen findet Ausdruck in formeller Bildung, in Kenntnissen der eigenen Rechte oder Kenntnissen kontextspezifischer Informationen. Diese können Akteure dazu befähigen, Zugang zu Land zu erlangen oder aufrechtzuhalten. Die Fähigkeit zu lesen, ist die Voraussetzung dafür, schriftliche Informationen, wie Ankündigungen zu einem geplanten Projekt, zu erhalten. Die Fähigkeit zu schreiben ist notwendig, um formelle Briefe oder Anträge verfassen zu können. Kenntnisse rechtlicher Rahmenbedingungen sind notwendig für die Aushandlung von Zugang zu Land, z.B. über die Neuinterpretation bestehender Regeln und Normen. Fachkenntnisse können eine Voraussetzung dafür sein, bestimmte Arbeiten, wie das Führen von Maschinen, leisten zu können. Darüber hinaus sind Kenntnisse kontextspezifischer Informationen zentral, um überhaupt auf einen möglichen eigenen Nachteil zu reagieren. Akteure die nichts über ein geplantes Projekt wissen, 
können auch die damit verbundenen negativen Auswirkungen für sich nicht realisieren und werden dementsprechend keine Gegenmaßnahmen ergreifen oder die Teilnahme an Entscheidungsprozessen einfordern. Indikatoren für Wissen als relevanten Zugangsmechanismus ist der Rückgriff von Akteuren auf spezifische Kenntnisse, um Zugang zu Land zu erlangen oder Verweise darauf, dass sie ihren Zugang zu Land aufgrund fehlender Informationen oder Kenntnisse verloren haben. Ein Beispiel, das den Rückgriff auf spezifische Kenntnisse veranschaulicht, sind Bestrebungen von Akteuren, Zugang zu Land über andere als die bisher genutzten Wege - z.B. über Gerichtsverfahren - zu erlangen. Aussagen von Akteuren zur Intransparenz der Landvergabe und dem daraus resultierenden Verlust des Lands können ein Hinweis auf den Indikator der fehlenden Informationen sein.

Soziale Zugehörigkeit ist darüber hinaus auch für die anderen relationalen und strukturellen Zugangsmechanismen - Netzwerke und soziale Beziehungen - bedeutsam. Die mit sozialer Zugehörigkeit verbundenen Handlungsmöglichkeiten und -grenzen - beeinflussen, welche sozialen Beziehungen Akteure eingehen und in welchen Netzwerken sie sich engagieren (können).

Netzwerke als relationaler und struktureller Mechanismus können auf vielfältige Weise relevant für den Zugang zu Land sein: Netzwerke ermöglichen es, gemeinsam Einfluss auf Debatten und Entscheidungsprozesse zu nehmen, Wissen zu teilen und sich gegenseitig zu unterstützen. Indikator für Netzwerke als Zugangsmechanismus ist, dass die Zugehörigkeit zu Netzwerken Auswirkungen auf den Zugang zu Land hat. Die Zugehörigkeit zu traditionellen Institutionen und damit verbundene Privilegien in Bezug auf Zugang zu Land ist ein Beispiel für diesen Indikator. Ein weiteres Beispiel ist die Mitgliedschaft in einem professionellen Netzwerk, das über spezifisches Wissen Zugang zu Land ermöglichen kann. Auch in Bezug auf Zugang zu Land über Arbeit spielen Netzwerke eine zentrale Rolle. Über die Mitgliedschaft in Netzwerken erlangen Akteure möglicherweise privilegierten Zugang $\mathrm{zu}$ Arbeit.

Der dritte relationale und strukturelle Zugangsmechanismus sind soziale Beziehungen. Indikator für diesen Zugangsmechanismus ist, dass Akteure Zugang zu Land maßgeblich über Beziehungen zu anderen Akteuren erlangen. Ein Beispiel ist die Möglichkeit migrantischer Landnutzer*innen Zugang zu Land zu erlangen, indem sie sich um eine gute Integration in die lokale Gemeinschaft bemühen. Ein weiteres Beispiel ist der Zusammenhang von Zugang zu Land über Arbeit und einer guten Beziehung zu 
Akteuren, die diesbezüglich eine gate keeper-Funktion einnehmen. Auch sharecropping-Vereinbarungen oder andere individuelle Vereinbarungen, die Zugang zu Land ermöglichen, sind Indikatoren für soziale Beziehungen als Zugangsmechanismus. Zugang zu Land über sharecropping veranschaulicht, wie sich relationale und strukturelle Zugangsmechanismen mit rechtebasierten Zugangsmechanismen überschneiden.

\section{Operationalisierung der sichtbaren, versteckten und unsichtbaren Macht}

In der Analyse meiner beiden Fälle untersuche ich, inwiefern sich sichtbare, versteckte und unsichtbare Machtverhältnisse zwischen den Konfliktakteuren auf die Kontrolle ihres Zugangs zu Land - operationalisiert mittels der unterschiedlichen Zugangsmechanismen - auswirken und inwiefern sich die Ausübung unterschiedlicher Machtformen auf die Entstehung und den Verlauf von Konflikten um Zugang zu Land auswirken. Zu diesem Zweck entwickle ich im Folgenden Indikatoren, anhand derer ich in der Analyse erkenne, dass es sich um eine der drei Machtformen handelt.

Die Analyse von Machtverhältnissen beschäftigt sich im Kern mit der Frage, wie es Akteuren gelingt, sich die Zustimmung von Gegenspieler*innen zu sichern. Um von Machtausübung sprechen zu können, müssen für die Analyse zwei zentrale Bedingungen erfüllt sein. Die erste Bedingung ist, dass eine Person (A) auf eine bestimmte Weise handelt oder bewusst nicht handelt. Die zweite Bedingung ist, dass aufgrund dieses Handelns eine zweite Person (B) etwas tut, das sie sonst nicht tun würde. »Tun« bezieht sich auf ein breites Verständnis, das auch »denken«, »wollen«, »akzeptieren« oder »fühlen« umfasst. A kann B allein durch ihr Handeln oder im Zusammenspiel mit anderen Faktoren dazu bringen, etwas zu tun, das B sonst nicht tun würde (Lukes 2005: 43). Damit wir von Machtausübung sprechen können, bedarf es immer der Möglichkeit eines kontrafaktischen Szenarios, also der Option, dass B sich in Abwesenheit des Handelns von A anders entscheiden würde.

Der zentrale Indikator für die Ausübung sichtbarer Macht ist ein Befehl, der Gehorsam findet. Ein »Befehl« kann eine Anweisung oder eine Entscheidung sein, die Konsequenzen nach sich zieht. »Gehorsam finden« kann bedeuten, dass Gegenspieler*innen nicht in der Lage sind, sich dem Befehl zu widersetzen, dass sie ihn widerstandslos akzeptieren oder sogar unterstützen, auch wenn er sich gegen ihre Interessen richtet. Ein Beispiel ist die Fähigkeit von Akteuren, alleinige Kontrolle über den Zugang zu Land aus- 
zuüben. Das bedeutet, diese können eigenständig die Entscheidung treffen, Land zu vergeben, auch gegen den Willen anderer Akteure, die nicht die Fähigkeit haben, sie daran zu hindern.

Versteckte Macht umfasst alle Formen der Kontrolle von A über B, mittels derer A sich die Zustimmung von B sichert. In Konflikten um Zugang bezieht sich die Ausübung versteckter Macht also auf die Kontrolle des Zugangs zu Land. Formen der Kontrolle sind vielfältig. Sie umfassen die Kontrolle von Informationen, den Einfluss auf institutionelle Verfahrensweisen und Partizipationsmöglichkeiten, aber auch die Androhung negativer Konsequenzen oder Gewalt. Ein zentraler Indikator für die Ausübung versteckter Macht ist Ausschluss. Ausschluss kann bedeuten, dass A entscheidet, Themen, die ihren eigenen Interessen schaden könnten, jedoch für B von Bedeutung sind, von der Agenda auszuschließen und nicht zu einem potentiellen Aushandlungsgegenstand zu machen. Das bedeutet B hat die Möglichkeit über x zu verhandeln, nicht aber über $y$. Darüber hinaus kann sich Ausschluss darauf beziehen, dass das Handeln von A die Möglichkeiten von B, Entscheidungen zu treffen, einschränkt. Wenn B beispielsweise keine Informationen darüber erhält, dass eine bestimmte Entscheidung zur Verhandlung steht, hat sie auch nicht die Möglichkeit an diesem Entscheidungsfindungsprozess teilzuhaben. Um diese Form der versteckten Macht zu identifizieren, ist es sinnvoll, die Ausgestaltung institutioneller Verfahrensweisen und die damit verbundenen Handlungsmöglichkeiten der Akteure in den Blick zu nehmen. Formen der Kontrolle können darüber hinaus Zwang, Einfluss, Autorität und Manipulation umfassen (Lukes 2005: 21). Der Indikator für die Ausübung versteckter Macht mittels Zwangs ist die Zustimmung aus Angst vor negativen Konsequenzen. Ein Beispiel dafür ist, dass A ihre eigenen Interessen entgegen derjenigen von B durchsetzen kann, indem sie B bei Widerstand mit Konsequenzen droht, die im Gegensatz zu ihren Interessen stehen. Der Indikator für die Ausübung versteckter Macht mittels Einflusses ist die Zustimmung von B aufgrund positiver Erwartungen. A übt Einfluss auf B aus, wenn sie sich den bereitwilligen Gehorsam von B sichert, ohne dafür implizit oder explizit negative Konsequenzen androhen zu müssen. Dies kann A beispielsweise gelingen, indem sie mögliche positive Auswirkungen der Entscheidung in den Mittelpunkt rückt oder B »Belohnung« für ihre Zustimmung in Aussicht stellt. Der Indikator für die Ausübung versteckter Macht mittels Autorität ist Anerkennung. A übt Autorität aus, wenn B ihr Handeln widerstandslos anerkennt, da sie es als angemessen hinsichtlich ihrer eigenen verinnerlichten Werte und Normen ansieht. Dies drückt sich beispielsweise anhand von Sätzen wie »Das 
war schon immer so« oder »So funktioniert das bei uns« aus. Indikator für die Ausübung versteckter Macht mittels Manipulation ist Zustimmung aufgrund fehlender Informationen. Ein Beispiel dafür ist ein Hinweis darauf, dass A in Bezug auf eine bestimmte Entscheidung B zentrale Informationen vorenthalten hat und B aufgrund dessen der Entscheidung zustimmt. In einem Szenario, in dem Landnutzer*innen ihr Land widerstandslos einem Unternehmen überlassen, in der Annahme, angemessen dafür entschädigt zu werden, ist es wahrscheinlich, dass sie anders gehandelt hätten, wenn sie über die unangemessene Höhe der Entschädigung informiert worden wären.

Die Ausübung unsichtbarer Macht bedeutet, andere so in ihren Einstellungen und Bedürfnissen zu beeinflussen, dass sie - indem sie zustimmen, einwilligen oder keinen Widerstand leisten - letztendlich entgegen ihrer ethischen Interessen handeln und willig an ihrer Unterdrückung mitwirken. Die Herausforderung, diese Form der Machtausübung empirisch zu analysieren, liegt darin, die ethischen Interessen der Akteure zu ermitteln.

Die Bestimmung von Interessen - sofern es sich nicht um manifeste Interessen handelt, die Akteure formulieren und/oder die sich aus ihren Handlungen ablesen lassen - ist grundsätzlich schwierig. Akteure haben in der Regel vielfältige unterschiedliche, teilweise widersprüchliche oder nicht miteinander vereinbare Interessen (Lukes 2005: 147). So können Interessen bezüglich des eigenen psychischen Wohlbefindens solchen gegenüberstehen, die sich auf das finanzielle Wohlergehen und die Sicherung eigener Grundbedürfnisse beziehen. Eine Person kann das Bedürfnis haben, im Sinne des psychischen Wohlbefindens mehr Erholungszeit für sich zu haben, bemüht sich aber dennoch um eine neue Arbeit mit höherem Stundenumfang, da sie ebenfalls ein Interesse daran hat, mehr zu verdienen. Wie ist es also möglich, empirisch die ethischen Interessen von Akteuren zu ermitteln, ohne a priori gesetzte Annahmen zu treffen und aus einer Außenperspektive den Akteuren Interessen zu unterstellen? Und wenn wir annehmen, dass Akteure vielfältige, auch widersprüchliche Interessen haben, welche dieser unterschiedlichen Interessen bestimmen wir dann letztendlich als die ethischen Interessen?

Die Außenperspektive der Forschenden in der Untersuchung unsichtbarer Machtverhältnisse ist in dem Konzept der unsichtbaren Macht selbst verankert. Die Annahme, dass die Zustimmung schwächerer Akteure zu ihrer Unterdrückung über die Einflussnahme auf ihre Einstellungen und Bedürfnisse sichergestellt wird, erfordert ein Urteilsvermögen, das sich von dem der vermeintlich betroffenen Akteure unterscheidet (Lukes 2005: 146). Denn die 
Ausübung unsichtbarer Macht beruht auf der Fähigkeit von A zu täuschen, und damit bei B falsche Annahmen zu erzeugen (ebd. : 149).

Für die Ermittlung ethischer Interessen bedarf es eines Referenzrahmens, der bestimmt, auf welchen Gegenstand sich die Untersuchung bezieht. Diesen Referenzrahmen setzt das Forschungsthema. Welche Interessen als ethische Interessen im Zentrum der Analyse stehen sollen und in welches Verhältnis unterschiedliche Interessen zueinander gesetzt werden, ergibt sich demnach aus der Perspektive der Forschenden. Sie setzt den Schwerpunkt der Untersuchung. In dieser Studie sind die ethischen Interessen von Akteuren in Bezug auf den Zugang zu Land zu ermitteln. Diesbezüglich stellt sich die Frage, inwiefern der Verlust von Zugang zu Land den Voraussetzungen für ein gutes Leben und somit den ethischen Interessen entgegensteht? Für die Operationalisierung ethischer Interessen nehme ich eine menschenrechtliche Perspektive ein. Insbesondere beziehe ich mich auf den Pakt über wirtschaftliche, soziale und kulturelle Rechte (United Nations General Assembly 1966). Demnach sind grundlegende Voraussetzungen für ein gutes Leben das Recht auf angemessene Entlohnung, auf angemessenen Lebensstandard, der sich über angemessene Nahrung, Kleidung und Wohnung ausdrückt, sowie das Recht auf Bildung. Diesbezüglich geht es mir nicht um eine normative Beurteilung, was als angemessen angesehen werden kann. Vielmehr gehe ich davon aus, dass die ethischen Interessen von Akteuren dann verletzt werden, wenn sich ihre Situation in Bezug auf diese Rechte im Verlauf der Landvergabe verschlechtert hat. Wenn eine Landnutzerin beschreibt, dass land grabbing dazu geführt hat, dass ihr Einkommen - im Gegensatz zu vorher - nicht mehr ausreicht, um genügend Lebensmittel zu kaufen oder ihren Kindern eine angemessene Bildung zu ermöglichen, deutet dies darauf hin, dass gegen ihre ethischen Interessen gehandelt wurde.

Aus Perspektive des Konzepts der unsichtbaren Macht beeinflusst die gesellschaftliche Ordnung die Bedürfnisse und Einstellungen von Akteuren und wirkt damit potenziell gegen deren ethische Interessen. Ein Ansatzpunkt, um die ethischen Interessen von Akteuren zu ermitteln, besteht darin $\mathrm{zu}$ bestimmen, was Akteure tun oder bevorzugen würden, wenn sie frei von gesellschaftlichen, strukturellen und wirtschaftlichen Zwängen entscheiden könnten (kontrafaktisches Handeln) (Lukes 2005: 38). Ethische Interessen sind nicht als »Tatsachen« zu denken, sondern als analytisches Hilfsmittel, um ein erklärendes Verstehen in einem bestimmten Sinnzusammenhang zu ermöglichen. Ihre Bestimmung dient nicht dazu, eine »Wahrheit« aufzudecken, sondern dazu zu zeigen, dass Akteure Interessen haben (können), 
für die sie nicht eintreten (können oder wollen), da sich ihnen aufgrund der gesellschaftlichen Ordnung, in der sie leben, oder aufgrund ihrer Sozialisation keine entsprechenden Handlungsmöglichkeiten bieten. Sie verhandeln daher nur Themen, die es bereits in Entscheidungsräume geschafft haben oder bezüglich derer sie Handlungsmöglichkeiten erkennen. Das bedeutet jedoch nicht, dass sich Akteure keiner anderen Interessen bewusst werden können, wenn sie sich beispielsweise in ein kontrafaktisches Szenario hineindenken oder eine Zukunftsvision entwickeln. Es bedeutet nur, dass sie ohne solche Vorstellungen bestimmte Interessen möglicherweise nicht formulieren, da sie sie als utopisch, unrealistisch und somit nicht handlungsrelevant erachten. Über die Bestimmung kontrafaktischen Handelns lassen sich so tieferliegende Interessen identifizieren. Denn Akteure kennen die Handlungsmöglichkeiten und -beschränkungen sowie die Konsequenzen von Handlungen, die mit bestimmten sozialen Strukturen verbunden sind. Abhängig von ihrer sozialen Position leiten sie daraus Gründe für ihr Handeln oder Nicht-Handeln ab und formulieren ihre Interessen entsprechend (Schuck 2014: 316). Indem Akteure sich also vorstellen, von einzelnen »Zwängen« befreit zu sein, können sie sich ihren tieferliegenden Interessen annähern. Darüber hinaus gilt zu berücksichtigen, dass gesellschaftliche Strukturen oder Sozialisation Einstellungen und Bedürfnisse eventuell bereits so beeinflusst haben, dass Akteure bestimmte Handlungsmöglichkeiten gar nicht in Erwägung ziehen.

In Konflikten um Zugang bedeutet die Ausübung unsichtbarer Macht, dass machtvolle Akteure Zugang zu Land beschränken können, ohne dass diejenigen, deren Zugang beschränkt wird, Widerstand leisten. Der fehlende Widerstand kann darin begründet liegen, dass Akteure keine Alternative sehen oder dass sie fälschlicherweise annehmen, die Entscheidung könne vorteilhaft für sie sein. Ein Indikator für die Ausübung versteckter Macht ist die Übernahme zentraler Argumente von stärkeren Akteuren durch die schwächeren Akteure und daraus resultierendes Handeln oder Nicht-Handeln entgegen der ethischen Interessen. Dies kann auf dominante Diskurse oder Sozialisationsprozesse zurückzuführen sein. Ein weiterer Indikator sind Hinweise auf Täuschung oder das Vortäuschen falscher Tatsachen. Ein Beispiel dafür könnte sein, dass Akteure eine bestimmte Entscheidung aufgrund vermeintlicher Vorteile für gut halten, diese Vorteile ihnen selbst jedoch nachweislich nicht zugutekommen. 
Tabelle 2: Operationalisierung der theoretischen Kategorien

\begin{tabular}{|c|c|}
\hline Kategorie & Indikatoren \\
\hline $\begin{array}{l}\text { Rechtebasierte } \\
\text { Zugangsmechanismen }\end{array}$ & $\begin{array}{l}\text { Akteure setzen legale und illegale Mittel ein, um Zugang zu } \\
\text { Land zu erlangen } \\
\text { Beispiele für legale Mittel: Besitzurkunden, Pachtverträge, } \\
\text { traditionelle Rechte, sharecropping-Vereinbarungen, Entschädi- } \\
\text { gungszahlungen etc. } \\
\text { Beispiele für illegale Mittel: Androhung oder Anwendung von } \\
\text { Gewalt, Diebstahl, Vortäuschen falscher Tatsachen etc. }\end{array}$ \\
\hline $\begin{array}{l}\text { Relationale und } \\
\text { strukturelle } \\
\text { Zugangsmechanismen }\end{array}$ & $\begin{array}{l}\text { Akteure beziehen sich auf ihre soziale Zugehörigkeit, um Zu- } \\
\text { gang zu Land zu erlangen } \\
\text { Beispiele für Kategorien sozialer Zugehörigkeit: Autochthonie, } \\
\text { first comer/late comer, Herkunft, Ceschlecht, Alter }\end{array}$ \\
\hline
\end{tabular}

Akteure interpretieren Landrechte zu ihren Gunsten

Beispiel: Akteure verhandeln ihre soziale Zugehörigkeit, um Zugang zu Land zu erlangen.

Akteure erlangen Zugang zu Land über die Mitgliedschaft in Netzwerken

Beispiele für Netzwerke: lokale Gemeinschaft, traditionelle Institutionen, professionelle Netzwerke, politische Netzwerke

Akteure erlangen oder kontrollieren Zugang zu Land aufgrund spezifischer Kenntnisse

Beispiele für spezifische Kenntnisse: Formelle Bildung, Kenntnisse der eigenen Rechte, Kenntnisse kontextspezifischer Informationen

Akteure erlangen Zugang zu Land aufgrund sozialer Beziehungen

Beispiele: Beziehung zu Personen, die Zugang zu Land kontrollieren, Integration von Migrant"innen in lokaler Cemeinschaft, Zugang zu Arbeit über Beziehungen etc. 


\begin{tabular}{|c|c|}
\hline Kategorie & Indikatoren \\
\hline Sichtbare Macht & $\begin{array}{l}\text { Befehl findet Gehorsam } \\
\text { Beispiel: A bringt B dazu etwas zu tun (denken, fühlen, wollen, } \\
\text { akzeptieren), auch wenn B etwas anderes vorziehen würde. }\end{array}$ \\
\hline Versteckte Macht & $\begin{array}{l}\text { Ausschluss von Akteuren oder Themen } \\
\text { Beispiele: } \\
\text { a)Institutionelle Verfahrensweisen verhindern die Herausforde- } \\
\text { rung etablierter Werte und Normen } \\
\text { b) Beschränkung der Verhandlung widersprüchlicher Interessen } \\
\text { durch den Ausschluss bestimmter Themen aus Entscheidungs- } \\
\text { räumen (Nicht-Entscheidungen) } \\
\text { c) Beschränkung von Entscheidungen durch Kontrolle von Infor- } \\
\text { mationen }\end{array}$ \\
\hline
\end{tabular}

Zustimmung aus Angst vor negativen Konsequenzen

Beispiel: B stimmt As Entscheidung zu, da sie aufgrund von As Androhung Angst hat.

Zustimmung aufgrund positiver Erwartungen

Beispiel: A verspricht $B$ für ihre Einwilligung eine Belohnung.

Zustimmung aufgrund fehlender Informationen

Beispiel: Bstimmt Entscheidung $x z u$, da sie Entscheidungy nicht als Alternative erkennt. 


\begin{tabular}{|c|c|}
\hline Kategorie & Indikatoren \\
\hline \multirow[t]{6}{*}{ Unsichtbare Macht } & $\begin{array}{l}\text { Schwächere Akteure übernehmen die Argumente der stärke- } \\
\text { ren Akteure }\end{array}$ \\
\hline & $\begin{array}{l}\text { Beispiel: A beeinflusst Bs Einstellungen und Absichten über die } \\
\text { Kontrolle von Informationen, Sozialisation (verinnerlichte Re- } \\
\text { geln und Normen etc.) und den Einfluss auf Diskurse. }\end{array}$ \\
\hline & Hinweise auf Täuschung \\
\hline & $\begin{array}{l}\text { Beispiel: B erklärt eine bestimmte Entscheidung aufgrund er- } \\
\text { warteter Vorteile, die ihm selbst jedoch nachweislich nicht zugu- } \\
\text { tekommen. }\end{array}$ \\
\hline & Anerkennung entgegen der eigenen Interessen \\
\hline & $\begin{array}{l}\text { Beispiel: B stimmt As Entscheidung entgegen ihrer Interessen } \\
\text { zu, da sie diese mit Blick auf verinnerlichte Werte und Normen } \\
\text { (Sozialisation) als richtig empfindet. }\end{array}$ \\
\hline
\end{tabular}


